Clio. Femmes, Genre, Histoire

1 | 1995

Résistances et Libérations France 1940-1945

\title{
Résistance et différence des sexes : bilan et perspectives
}

Paula SCHWARTZ

\section{(2) OpenEdition}

1 Journals

Édition électronique

URL : https://journals.openedition.org/clio/516

DOI : 10.4000/clio.516

ISSN : 1777-5299

Éditeur

Belin

Édition imprimée

Date de publication : 1 avril 1995

ISSN : 1252-7017

Référence électronique

Paula SCHWARTZ, "Résistance et différence des sexes : bilan et perspectives », Clio. Femmes, Genre, Histoire [En ligne], 1 | 1995, mis en ligne le 01 janvier 2005, consulté le 23 avril 2022. URL : http:// journals.openedition.org/clio/516 ; DOI : https://doi.org/10.4000/clio.516

Ce document a été généré automatiquement le 23 avril 2022

Tous droits réservés 


\title{
Résistance et différence des sexes : bilan et perspectives
}

\author{
Paula SCHWARTZ
}

1 Histoire des femmes. Histoire des femmes dans la Résistance. Histoire des femmes communistes dans la Résistance ${ }^{1}$. Sujets de recherche faits sur mesure pour faire horreur à tout public américain. Du moins ce fut le présupposé des Français, puisque bon nombre d'entre vous m'ont demandé, avec étonnement, quelle réaction un tel projet d'études provoquait outre-Atlantique, en terre ennemie des «rouges». Et pourtant, à ma grande surprise, le premier scepticisme à vaincre aux États-Unis il y a quinze ans, lorsque j'entreprenais mes premières recherches, fut celui suscité à l'égard de l'histoire des femmes. Encore plus suspect que le communisme pour les Américains fut l'idée de travailler sur les femmes, tandis qu'en France le problème se posait tout autrement. Pour certains Français, l'étude d'un aspect du communisme semblait somme toute légitime et n'excluait pas forcément une sympathie présumée de la part du chercheur. Mais curieusement, cette question ne se posait pas aux États-Unis, du moins dans un premier temps, tant la possibilité d'un parti pris communisant était, sinon exclue, du moins improbable. Cependant, aux États-Unis à la fin des années soixante-dix, enquêter sur les femmes impliquait d'autres a priori douteux : celui de la différence, de l'engagement féministe, voire de quelque triomphalisme amazonien (!).

2 Les enjeux d'une approche axée sur les femmes ne sont plus les mêmes depuis que l'histoire des femmes, et plus récemment la notion de la différence des sexes comme catégorie valable d'analyse, ont fait tout bonnement leur chemin... en brûlant les étapes, comme en témoigne la véritable profusion d'ouvrages, d'études, de séminaires, de mémoires parus ces dix dernières années. Naturellement la publication ne constitue pas à elle seule l'indice d'une insertion parfaite, ni dans le monde scientifique, ni chez le grand public, mais elle suggère tout de même une percée non négligeable dans le marché des connaissances. Les bases d'une légitimité relative de l'histoire des femmes sont dorénavant acquises. Il reste maintenant à poursuivre l'interrogation sur la construction des rôles masculins et féminins, et sur le déploiement et la signification de 
ces rôles dans un champ social et politique donné : celui, par exemple, de la Résistance antifasciste en France pendant la Deuxième Guerre mondiale.

3 Le problème de la différence des sexes comme vecteur d'analyse de la Résistance soulève bien des questions. Dans quelle mesure les représentations diverses de la Résistance - qu'elles soient écrites, orales, iconographiques, ou cérémoniales - se prêtent-elles à l'interrogation de leur caractère sexué ? En ce moment charnière du cinquantième anniversaire de la Libération, comment le profil historiographique des femmes se dessine-t-il, et implicitement ou explicitement, celui du rapport social des sexes pendant la Résistance? Enfin, quelles perspectives s'ouvrent à l'horizon? Avant d'esquisser quelques réponses provisoires aux questions qui nous concernent ici, les enjeux de notre appareil conceptuel et discursif méritent quelques commentaires.

Regard sexué, différence des sexes, gender : nous sommes toujours à la recherche d'un langage commun qui exprime la spécificité de la notion de «l'inscription de la différence sociale sur le corps sexué », selon l'historienne Joan W. Scott ${ }^{2}$. Quoique la différence existe incontestablement au niveau du corps, la façon dont elle est vue, vécue, et valorisée, relève du social. C'est cet ensemble d'idées qu'on revendique par l'usage du terme "gender» en anglais, un terme qui malheureusement se prête mal à l'usage français, son équivalent le plus proche ayant des résonances multiples. Il nous faut toutefois un vocabulaire qui tient compte de la distinction entre l'être biologique et l'être social, bien que la question de leur relation dans telle ou telle circonstance reste largement ouverte. Si « gender » tend de plus en plus à remplacer «femmes » dans l'historiographie actuelle, c'est non seulement pour intégrer les femmes dans une réalité plus complexe, mais pour éviter de poser l'Homme (blanc, occidental, de classe moyenne en l'occurrence) comme le repère par excellence vis à vis duquel on situe cette Autre.

5 La question du langage relève donc de la méthode autant que de la sémantique, car les formules telles que « différence des sexes » et « regard sexué » renvoient justement à la fatalité biologique, comme si les comportements sociaux, les relations humaines, et leurs significations à travers le temps et l'espace étaient déterminés au préalable par le seul corps. Recourir à ces formules sans condition tend à réinscrire la différence comme si elle allait de soi : ce serait saboter le projet par son propre discours. Si nous disons « différence des sexes » ou "regard sexué » par respect des usages en vigueur, c'est sous toute réserve et en précisant que la notion telle que nous l'entendons ici dépasse largement le domaine du sexe biologique.

6 Mais les difficultés d'une telle démarche ne se limitent pas aux leurres linguistiques. Par souci de reconnaître la complexité des relations humaines dans toute leur ampleur - et peut-être aussi, pour certains, d'éviter de nommer les femmes explicitement comme objet d'études - on risque de se retrouver à la case départ par une réoccultation, voulue ou non, des femmes. D'où le débat qui partage les partisans des women's studies, qui très souvent impliquent la prise en compte de la notion de gender, et ceux qui préfèrent sa version refondue et rééquilibrée - gender studies -, qui à la fois englobe et dépasse l'étude des femmes proprement dite. Quoiqu'il en soit, chercher la femme dans l'histoire de la Résistance, sans remettre en question les notions même de " femme » et de "résistance », risque une réécriture du passé qui ne fait que doubler l'histoire officielle, alors que ne voir la femme que par rapport à l'homme risque d'occulter ce qui est spécifique à son statut, à ses ressources, à son comportement. Une esquisse schématique d'un demi-siècle d'historiographie portant sur la Résistance et les 
femmes démontre l'importance et la complémentarité de ces deux approches - en dépit de, et à cause de - leurs insuffisances respectives.

7 Histoires de femmes, histoire de la Résistance

8 Au lendemain de la Libération sont parus divers ouvrages en forme de mémoires, de brochures commémoratives, de livres d'histoire consacrés à la Résistance, dont un certain nombre évoque le rôle des femmes, soit explicitement, soit par allusion ${ }^{3}$. Mais cette production est devancée de loin par une littérature historiographique et mémoriale à dominante masculine. Si la présence des femmes se tisse en filigrane à travers la mémoire collective et individuelle, toujours est-il qu'elles figurent rarement comme objet de recherche à proprement parler ${ }^{4}$. L'exception importante est celle de l'historiographie communiste, où l'évocation du rôle des femmes au sein du mouvement national de Résistance sert à asseoir une certaine légitimité politique dès la fin de la guerre ${ }^{5}$.

9 C'est la conjoncture des années soixante-dix qui marque un tournant majeur : au mouvement des femmes, dont l'impact sur toutes les sciences humaines s'est fait sentir, s'est ajoutée la «nouvelle histoire », porteuse d'un projet de récupérer les laissés-pourcompte de l'histoire érudite. Dorénavant sont privilégiées la longue durée au lieu de l'événement, les mentalités à la place des grandes idées, l'histoire sociale " d'en bas » à la place de l'histoire politique des élites. Le croisement du mouvement des femmes, qui a entraîné une prise de conscience et un renouveau de la théorie et de la critique féministes, des nouvelles tendances historiographiques de l'autre, a suscité un regain d'intérêt pour l'histoire des femmes en général, et plus pertinemment pour les questions qui nous intéressent ici, celle de la Résistance ${ }^{6}$.

10 Fallait-il attendre cette prise de conscience de soi-même en tant que femme, et en tant que sujet d'histoire, pour que les anciennes résistantes prennent la plume ? La parution d'un corpus impressionnant de mémoires à partir des années quatre-vingt nous le laisse croire, car un certain nombre d'entre elles commencent à décrire, dans leurs propres mots et selon leur propre logique, leur parcours résistant ${ }^{7}$. Les unes attribuent ce geste autobiographique tardif à la disponibilité de temps et d'esprit qui accompagne la retraite de la vie active. D'autres citent le déclin des obligations familiales, ou encore la présence d'une troisième génération plus curieuse (ou moins discrète) que ses parents... Quoiqu'il en soit, d'une occultation quasi-opaque à une place de plus en plus assurée dans l'historiographie, dans le discours politique actuel, dans le rituel cérémonial et commémoratif de la Résistance, bref dans les cultures et cultes de la Résistance, les femmes sont dorénavant sur scène.

11 La mise en valeur d'un héroïsme au féminin, qu'elle soit affichée ou sous-entendue, a longtemps caractérise une bonne partie de la littérature sur les femmes résistantes ${ }^{8}$. Peut-être fut-il souhaitable, dans un premier temps, de constituer un palmarès de femmes qui se détachent de la masse par leur exemplarité et par leurs souffrances. De toute façon, des femmes comme Marie-Madeleine Fourcade ${ }^{9}$, Berty Albrecht ${ }^{10}$, et Danièle Casanova ${ }^{11} \mathrm{y}$ figurent à titre emblématique, car elles sont censées représenter la participation féminine dans son ensemble. Mais faute de mettre en cause la catégorie même de "résistance", d'interroger à nouveau ce que nous entendons par "héroïsme» et "exemplarité ", cette approche risque de reproduire l'histoire officielle, voire masculine. Dans beaucoup de cas, les femmes sont identifiées comme exemplaires dans la mesure où elles ressemblent aux hommes: les dirigeantes qui occupaient des postes clefs au sein de leurs organisations, les partisanes qui ont 
combattu les armes à la main à l'image de leurs soldats-« frères ». Une telle approche tend à renforcer une notion valable mais trop partielle de ce qui constitue la « résistance $»^{12}$.

Comme pour corriger cette tendance, l'historiographie des années soixante-dix se distingue par son souci de délimiter ce qui est spécifique à la résistance au féminin. Ce sont les femmes «ordinaires » dans leur ensemble, beaucoup plus que les individus exemplaires, qui retiennent l'attention. De ces études ressortent d'une part la différence entre hommes et femmes en ce qui concerne la distribution des rôles, et de l'autre la singularité d'une organisation et d'une presse clandestines à l'intention explicite des femmes, mises en place par le Parti communiste ${ }^{13}$. Les itinéraires de femmes résistantes sont saisissants à cet égard, car ils mettent en évidence la particularité du recrutement, des formes de la militance, des peines encourues, pour ne citer que les différences les plus éclatantes. Cependant, mettre l'accent sur la spécificité de l'expérience et de l'organisation féminines risque d'induire à un certain «essentialisme », selon lequel une nature féminine innée est supposée expliquer des phénomènes qui relèvent plutôt du social.

13 L'image classique de la femme résistante incline à réinscrire cette notion sexuée de la différence. Si les femmes individuelles sont parfois difficiles à traquer dans les archives, un certain comportement "féminin " se dégage aisément de maints témoignages, récits, et mémoires. L'exploitation des attentes, des conventions sociales, et du statu quo était l'apanage de tout résistant. Mais hommes et femmes ne se servaient pas toujours des mêmes armes. C'est précisément le système d'auto-défense des femmes qui caractérise les images d'Epinal de la résistance au féminin : la pourvoyeuse d'armes déguisée en "ménagère » qui transporte sa contrebande dans un panier à l'ombre des poireaux; l'agent de liaison déguisée en «mère » qui dissimule son matériel sur son ventre en la forme d'une présumée grossesse ; la clandestine déguisée en « jeune fille » qui fait du flirt pour circuler à travers contrôles et barrages; enfin une mère (une vraie) qui promène un bébé (un vrai) dont la couche recouvre des cartouches.

14 Autant de ruses propres à la femme qu'il convient de confronter à d'autres stratagèmes axés sur la différence des sexes, et pratiqués aussi bien par des résistantes que par leur pairs masculins : les noms de guerre, de sexe volontairement ambigu, aussi bien que le travestisme, ont servi aux hommes comme aux femmes pour brouiller les identités ${ }^{14}$. Une analyse axée sur la différence des sexes permet donc de voir ces subterfuges comme des phénomènes issus d'un social ambiant et non de quelque disposition somme toute « naturelle. »

15 La tendance à voir dans la pratique résistante une certaine qualité typiquement " féminine » explique, du moins en partie, l'absence d'une historiographie pour celles qui se sont rangées du côté de Vichy. La notion de femmes collaboratrices, auteurs de crimes ou de dénonciations, coupables de complicités anti-« françaises » porte atteinte à des idées profondément ancrées dans nos mentalités collectives ${ }^{15}$. Il a fallu un certain temps pour aborder le problème de la femme fasciste ou fascisante dans les pays belligérants. Les travaux de Claudia Koonz et de Victoria De Grazia, qui traitent l'action des femmes dans toute sa complexité, sont instructifs à cet égard ${ }^{16}$. Pour l'historienne aussi bien que pour son public, la notion de la militante fasciste ébranle l'archétype réconfortant de la femme nourricière et foncièrement pacifique. Alors que de telles réticences ont été vaincues dans l'historiographie de l'Allemagne nazie et de l'Italie fasciste, il n'en était pas de même pour la France dont le passé ambigu n'a connu de 
véritable mise au point que relativement tard, y compris en ce qui concerne l'histoire des femmes ${ }^{17}$.

d'acteurs historiques considérés alors comme marginaux, sans lesquels la Résistance
dans toute son ampleur et dans toute son originalité se trouve diminuée. Le défi n'était
pas sans risques. Si les premiers pas ont été franchis avec beaucoup de succès, toujours faut-il poser la question de la construction même d'un objet de recherche qui s'avère fuyant à plusieurs égards.

18 Tout d'abord une analyse qui tient compte de la dimension sexuée dans les formes et fonctionnement de la Résistance repose au préalable sur une histoire des femmes. Or, s'il n'est plus question d'insister sur l'omniprésence des femmes dans le mouvement (même si d'autres «résistances " perdurent de la part des historiens ${ }^{18}$ ), il nous reste à intégrer l'histoire des femmes dans une problématique plus large. Mais d'importants obstacles d'ordre conceptuel et pratique minent le terrain.

La mise en cause des catégories d'analyse est d'autant plus importante que l'objet de recherches se dérobe à l'œil nu. C'est le cas de tout clandestin, et tout particulièrement celui des femmes, dont l'invisibilité relative pendant la guerre se prolonge, sous des formes différentes, jusqu'à nos jours. Les noms de guerre féminins, masculins, ou de sexe indéterminé (tels "Claude», ou bien "Dominique») servaient à brouiller l'identité et souvent le sexe d'un agent souterrain ${ }^{19}$. Mais les femmes sont cachées à plusieurs niveaux. Aux noms de guerre de la résistante s'ajoutent son nom de jeune fille, et encore, le cas échéant, son ou ses noms de femme mariée. Même en temps "normal », l'homme porte son nom du berceau au tombeau, tandis que la femme a souvent des identités superposées qui correspondent à son état civil - la femme serait dans ce sens toujours en quelque sorte une clandestine. Il se pouvait qu'une résistante mariée, une fois arrêtée et détenue, soit écrouée dans les registres sous son nom de jeune fille, ce qui rend les rapprochements et les repérages d'autant plus difficiles.

20 D'autres exemples se multiplient à volonté: Christiane Charua ne porte plus son prénom d'origine, ni même son nom de famille d'avant-guerre. Résistante, déportée à Auschwitz dans le convoi des 31 000, elle garde son nom de guerre - en souvenir - après sa libération et ensuite se remarie, si bien qu'elle est connue aujourd'hui comme Cécile Borras $^{20}$. De même pour la résistante Marie-Jo Chombart de Lauwe, qui ne s'appelait ni Marie-Jo (son nom de guerre adoptif, adopté à tout jamais), ni Chombart de Lauwe (son nom de mariée) à l'origine. Chercher la femme comme objet d'histoire, de la clandestinité à la lumière du jour, à travers les aléas de la guerre et de l'amour, n'est pas toujours chose facile.

21 Aux problèmes d'ordre pratique s'ajoutent d'autres problèmes d'ordre plus épistémologique. Ce qu'on voit dans les documents est non seulement tributaire de ses propres optique et catégories d'analyse, mais aussi de celles de ses sources. Trouver les femmes résistantes implique une méthode qui permet de contourner le parti pris des sources primaires. Tout d'abord, "les femmes" ne constituent que rarement une catégorie à part dans les catalogues, répertoires, ou fichiers des archives et bibliothèques $^{21}$. Et pour dégager celles qui ont milité dans des organisations communistes, il a fallu une stratégie de recherche qui consistait à les "voir» autrement. Elles se dissimulaient sous des rubriques globalisantes - telles «les jeunes » et même « les masses » - ou bien réductrices, comme celle de «ménagères ». De toute 
une politique de la différence découle une taxinomie bien spéciale qu'il faut reconnaître pour enfin cerner les femmes.

Il est normal que le classement des documents d'époque se calque sur les catégories qui ont servi à l'origine à constituer ces mêmes documents. Un exemple démontre bien la façon dont les catégories qui ont prévalu pendant la guerre servent à structurer dans un premier temps les documents, et ensuite leur rangement, si bien qu'une certaine notion de la femme se glisse inaperçue même sous le regard avisé du chercheur. La politique du Parti communiste français (PCF) envers les femmes faisait partie d'une autre, encore plus vaste, qui visait à regrouper tous les éléments de la population au sein du Front national. Des comités étaient créés pour encadrer commerçants, cheminots, enseignants, paysans, intellectuels, étudiants, ouvriers de toutes sortes... et femmes. Parmi des classifications socio-professionnelles se range maladroitement celle des femmes, alors qu'être femme n'est pas plus un métier qu'un label socioéconomique. C'est universaliser la femme comme type, comme si toutes partageaient des intérêts, mobiles et qualifications identiques qui l'emportaient sur les différences de classe, de formation, de religion, de génération, ou d'orientation politique. Cette mentalité n'était pas le propre des seuls communistes, même si le PCF était la seule formation de la Résistance à s'adresser aux femmes en leur qualité de mères, d'épouses, de responsables du foyer.

Un contexte sexué

Si on peut parler de tâches, de stratégies, de comportements sexués pendant la Résistance, c'est que femmes et hommes - qu'ils aient été clandestins ou non, collaborateurs ou occupants, Français ou Allemands - figuraient sur une scène hautement structurée au préalable par la différence des sexes. Le fascisme en Italie, le nazisme en Allemagne, le régime de Vichy, ont tous partagé, à des degrés différents, un système d'idées et une politique d'État calqués sur la différence des sexes ${ }^{22}$. L'idéologie et la pratique des fascismes axées sur la différence biologique « raciale » et sexuelle ${ }^{23}$, le contexte social et politique de la vie quotidienne d'avant-guerre, l'appareil répressif mis en place par les autorités - de la traque des militants aux dispositifs policiers et judiciaires - concourent à l'évolution des moyens de lutte adaptés à la société ambiante. Quoi de plus normal qu'un mouvement clandestin soit le reflet, quoiqu'imparfait, de l'environnement social dont il est issu ? Quoi d'étonnant que les formes et moyens de la lutte aient un caractère sexué, lorsque la différence entre les femmes et les hommes constitue un des axes transversaux de l'univers social et politique dans lequel ils opèrent?

Que la différence des sexes ait fonctionné pendant la Résistance s'explique donc, en partie au moins, par son contexte. Signalons tout d'abord l'importance capitale des réseaux associatifs, amicaux, socio-professionnels, ou familiaux d'avant-guerre. Sympathisants et militants ont trouvé la voie de la Résistance organisée grâce, en grande partie, aux structures politiques et parapolitiques existantes. On a insisté à juste titre sur le rôle des réseaux socio-professionnels dans le recrutement, surtout en ce qui concerne les hommes ${ }^{24}$. Mais l'importance des attaches familiales et conjugales saute aux yeux lorsqu'on remonte le fil des parcours féminins ${ }^{25}$.

26 A ce propos le mouvement des Jeunes filles de France, fondé en 1936 comme l'organisation-sœur des Jeunesses communistes masculines, est doublement révélateur. Des jeunes militantes du JFF furent acheminées dans les rangs du parti résistant qui recrutait ses agents de liaison parmi ces éléments jeunes, sûrs, et féminins. Bien que cet 
organisation ait fourni à ses adhérentes une formation politique, elle revêtait aussi une fonction sociale, voire libératrice. Un des attraits nombreux des JFF, selon d'anciennes adhérentes des années trente, c'est qu'elle leur a permis une certaine indépendance, hors des contraintes qui soumettaient alors les jeunes filles à la stricte vigilance. Quelques anciennes des JFF qui ont milité plus tard dans la Résistance, avouent que leurs parents les autorisaient à sortir ou à partir en excursion sous l'égide du groupe, alors qu'autrement il n'en était pas question. Cela en dit long à la fois sur le rôle des mœurs sociales et l'apprentissage inégal des femmes à la vie politique.

Les résistants qui n'ont pas échappé à la répression ont souffert dans un univers judiciaire et carcéral structuré lui aussi par la différence des sexes. Pour combattre ses adversaires, les autorités françaises et allemandes ont parfois exercé une politique de répression différenciée selon le sexe du présumé coupable : peines et condamnations différentes, prisons et camps réservés aux hommes ou aux femmes. Face à une répression qui distinguait dans certaines circonstances entre hommes et femmes, les résistants ont forgé leurs formes de lutte spécifiques ; face aux résistants, les autorités ont façonné et refaçonné leurs méthodes, si bien que ces formes de lutte réciproques revêtaient elles aussi un caractère sexué ${ }^{26}$. Mais si on peut dire que la parenthèse de la guerre et de l'Occupation a favorisé une certaine expérimentation au niveau des rôles sexués, les structures institutionnelles et mentales n'ont pas toujours suivi le mouvement au même rythme.

Perspectives : mémoires et représentations

Tout comme les rapports entre les hommes et les femmes, la Résistance est fondamentalement et par excellence relationnelle : elle n'existe qu'« en fonction de »; elle ne se définit que " par rapport à ». Elle tient sa raison d'être de la seule existence d'une opposition. Dans ce sens, elle est destinée à œuvrer à sa propre disparition. Ce qui explique l'impulsion continue de la part de l'État, des historiens, et des créateurs d'après-guerre, de la faire revivre dans la mémoire.

Cette mémoire habite les représentations de la Résistance, d'où l'on constate qu'un sexe figuré peut être attribué aux choses aussi bien qu'aux personnes. Il n'est pas sans intérêt que la Résistance figure en la forme d'une femme dans Le Silence de la mer, ce récit issu de la clandestinité et un véritable acte de résistance en lui-même ${ }^{27}$. Au devant de la scène se situe un jeune officier allemand qui s'impose de force, occupation oblige, chez l'habitant français et sa nièce. La jeune femme reste muette jusqu'au dénouement de l'histoire, refusant obstinément de lui adresser la parole. A l'intérieur de ce récit en est un autre, raconté par l'occupant à ses deux hôtes. C'est l'histoire d'une femme (la France) qui résiste à son cavalier admiratif et enthousiaste (l'Allemagne). Faute de la séduire, il la prend de force pour réaliser son rêve de l'union des deux pays. Comme des poupées russes qui se rangent l'une dans l'autre, Le Silence de la mer abrite un récit en abîme qui lui ressemble. Dans les deux, la Résistance est française et elle est femme. Elle ne s'oppose pas de front, comme le ferait une armée légitime au découvert; elle est la plus faible devant la puissance supérieure que possède son adversaire masculin ; elle ne cède jamais malgré son déchirement intérieur. Il y a là des qualités dites "féminines " pour dépeindre un mouvement considéré à d'autres moments comme « viril $»^{28}$.

Que la Résistance soit une affaire d'hommes est suggéré par des représentations cinématographiques comme Le Chagrin et la Pitié (1971) de Marcel Ophuls ou Une Affaire de femmes (1988) de Claude Chabrol ${ }^{29}$. La culpabilité, le mensonge, la cupidité, et l'ignorance, tous symptômes d'une société profondément perturbée, sont habilement 
mis en scène par des figurantes ${ }^{30}$. Ces exemples, pris parmi d'autres, montrent que les représentations sexuées ne sont pas forcément immuables dans le temps et dans l'espace. Aussi faut-il toujours préciser comment les notions même de « résistance ", de « collaboration, » de «patrie » ou de «nation » sont représentées de façon sexuée dans l'imaginaire collectif ou individuel. Et chercher quels sont les enjeux politiques et culturels de telles représentations dans une situation donnée? Ce sont des pistes prometteuses pour une histoire des histoires des femmes dans la Résistance.

Virginia Woolf pose de manière aiguë la question de la relation de la femme, marginalisée et exclue de la vie civique, à l'État et à la politique. Dans son manifeste Three Guineas, écrit à l'aube de la Deuxième Guerre mondiale, Woolf affirme que l'exclusion des femmes de la sphère dite "publique » les rend particulièrement aptes à faire barrage au fascisme ${ }^{31}$. Les "filles d'hommes cultivés", constate-t-elle, sont dépourvues d'indépendance financière, exclues des universités et des professions, et écartées d'une participation civique à part entière, malgré dix-neuf ans de suffrage féminin. Par conséquent, ces femmes ne sont ni impliquées, ni investies dans la vie civique de la même façon que leurs frères. Elles sont donc en mesure de profiter de leur position marginale pour reconnaître, dénoncer, et combattre le fascisme dans toutes ses formes.

Si on poursuit le raisonnement de Woolf, et si l'on considère le suffrage comme la condition sine qua non de la citoyenneté, les Françaises seraient encore plus inclines à prendre position. Après tout, elles n'étaient ni électrices ni éligibles avant 1944. Leur situation inégale ne s'est nullement traduite par un manque d'engagement résistant. $\mathrm{Au}$ contraire, un des grands paradoxes concernant la participation des femmes à la Résistance, c'est qu'elles ont assumé leurs devoirs de citoyennes longtemps avant de pouvoir jouir de leurs droits ${ }^{32}$. Elles ont pris position, certes, mais au nom de quoi ?

D'où la question de l'identité nationale des femmes par rapport à celle de leurs compatriotes masculins. Comment les femmes françaises de cette époque vivaient-elles leur relation à la nation? Comment ont-elles concilié leur marginalisation par toutes les républiques qui ont précédé "l'État français " de Vichy ${ }^{33}$, avec un engagement résistant qui se voulait une défense de la patrie? Dans quel langage expriment-elles leurs mobiles, leurs actions, leurs buts ? Quoiqu'il en soit, on est en droit de s'attendre à une relation complexe et nuancée vis-à-vis de ce qu'on nomme "la France " (laquelle?). Et vraisemblablement le discours féminin, s'il y en a un, invoque un ensemble de valeurs et d'identités axées sur le social, la politique, le professionnel, la nation, et l'universel... mais à quel moments et dans quelles proportions ? Autrement dit, comment l'identité, qu'elle soit nationale ou autre, figure-t-il dans l'engagement des femmes résistantes, et dans la représentation de leur expérience résistante par la suite ? Dans quelle mesure la notion de la différence des sexes est-elle utile pour faire ressortir la complexité de l'engagement résistant?

La question d'identité soulève à son tour celle de la mémoire. L'époque que nous vivons est un moment privilégié pour toute recherche sur la période de la guerre. Nous sommes actuellement à la convergence de deux échéances dont la première annonce un commencement, la seconde une fin: les archives s'ouvrent davantage alors que la génération de la Résistance, qui nous a marqués et nous marque encore, s'éteint tout doucement. Le travail à l'avenir, comme celui qui s'amorce à l'heure actuelle, consistera à étudier le témoignage en sa qualité de texte dont la forme et les mots, les choix et les associations d'idées, les silences et les refoulements, ont une signification. 
Bref, il faudra désormais interroger les témoignages comme les historiens jusqu'à présent ont interrogé les témoins. La différence des sexes contribuera alors comme catégorie d'analyse à mettre en valeur tout témoignage, dans sa particularité aussi bien que dans son universalité, qu'il soit de provenance orale ou écrite ${ }^{34}$. A la place de l'histoire orale, dont la pratique sera un jour périmée par la force des choses, l'histoire de la Résistance se renouvellera aussi par des études comparatives. Arrive le moment où il sera envisageable d'évaluer la différence des sexes par rapport à d'autres critères, dans des circonstances comparables, par une analyse plus européenne des Résistances nationales.

A l'historien de la Résistance il incombe non seulement de déjouer les identités multiples de la femme - qu'elles soient inventées, imposées, ou revendiquées - mais aussi d'interroger son insertion dans un large contexte sexué. La notion de la différence des sexes et les problématiques qu'elle implique sont porteuses d'enseignements inestimables pour l'histoire des femmes, l'histoire de la Résistance, et l'histoire tout court, y compris l'histoire que nous vivons. Si rôles, stratagèmes, et représentations sont construits en fonction de la différence des sexes, il n'est pas impensable qu'ils se prêtent à une déconstruction éventuelle, à la fois dans l'histoire que nous écrivons, et dans l'histoire que nous fabriquons, consciemment ou à notre insu, à tort ou à raison, au jour le jour de notre existence collective.

\section{NOTES}

1. Ce texte a bénéficié des lectures, relectures, et conseils de Janet Horne, Nancy Rabinowitz, et Françoise Thébaud ; qu'elles en soient chaleureusement remerciées. 2. Joan Wallach Scott, Gender and the Politics of History, New York, Columbia University Press, 1988, p. 32. Ce livre, qui a fourni le cadre d'analyse pour cet article, a jeté les bases de toute réflexion sur les limites conceptuelles de l'histoire des femmes (women's history) et l'aspect heuristique de la notion de gender.

3. Parmi les ouvrages les plus précoces qui traitent explicitement le rôle des femmes, citons Elisabeth Terrenoire, Combattantes sans uniformes, Paris, Bloud et Gay, 1946 ; Edith Thomas, Cinq parmi d'autres, Paris, Editions de Minuit, 1947 ; Agnès Humbert, Notre guerre, Paris, Emile-Paul Frères, 1946 ; Louis Saurel, Les Femmes héroïques de la Résistance: Bertie Albrecht et Danièle Casanova, Paris, Nathan, 1945.

4. Voir par exemple les monographies qui ont fait date dans l'historiographie universitaire de la Résistance : Madeleine Baudoin, Histoire des Groupes Francs (M.U.R.) des Bouches-du Rhône de septembre 1943 à la Libération, Paris, Presses Universitaires de France, 1962 ; Marie Granet et Henri Michel, Combat: Histoire d'un mouvement de Résistance de juillet 1940 à juillet 1943, Paris, Presses Universitaires de France, 1957 ; Dominique Veillon, Le Franc-Tireur : un journal clandestin, un mouvement de Résistance, 1940-1944, Paris, Flammarion, 1977.

5. A titre d'exemple, voir les brochures commémoratives diverses éditées par l'Union des femmes françaises au sortir de la guerre, dont le Livre d'or dédié aux femmes héroïques mortes pour que vive la France (5 volumes), Paris, Imprimerie Centrale commerciale, 1951. 
6. Simone Bertrand, Mille visages, un seul combat, Paris, Les Editeurs Français Réunis, 1965 et Visages de dauphinoises, Grenoble, Imprimerie du Centre Régional de Recherche et de Documentation Pédagogiques, 1974. Voir aussi Nicole Chatel, Des Femmes dans la Résistance, Paris, Julliard, 1972 ; Ania Francos, Il était des femmes dans la Résistance, Paris, Stock, 1978 ; Marianne Monestier, Elles étaient cent et mille, Paris, Fayard, 1972. Les actes du colloque organisé par l'Union des femmes françaises et édités dans le livre collectif, Les femmes dans la Résistance, Paris, Editions du Rocher, 1977, méritent une mention à part.

7. Lucie Aubrac, Ils partiront dans l'ivresse, Paris, Editions du Seuil, 1984 ; Célia Bertin, Femmes sous l'occupation, Paris, Editions Stock, 1993 ; France Hamelin, Les Crayons de couleur, Paris, publié à compte d'auteur, 1982 et Femmes dans la nuit, 1939-1944, Paris, Renaudot et Cie, 1988 ; Liliane Lévy-Osbert, Jeunesse vers l'abîme, Paris, Etudes et documents internationaux, 1992 ; Lise London, L'écheveau du temps, la mégère de la rue Daguerre, souvenirs de Résistance, Paris, Editions du Seuil, 1995 ; Cécile OuzouliasRomagon, J'étais agent de liaison FTPF, Paris, Editions Messidor, 1988. Signalons également Edith Thomas, Le Témoin compromis, mémoires et Pages de journal 1939-1944, les deux présentés par Dorothy Kaufmann et parus chez Viviane Hamy, 1995.

8. A titre d'exemple voir Margaret L. Rossiter, Women in the Resistance, New York, Praeger Publishers, 1986.

9. Marie-Madeleine Fourcade, L'Arche de Noë, Paris, Fayard, 1968.

10. Mireille Albrecht, Berty : la grande figure féminine de la Résistance, Paris, Editions Robert Laffont, 1986.

11. Pierre Durand, Danielle Casanova, l'indomptable, Paris, Editions Messidor, 1990. Avec Marie-Claude Vaillant-Couturier, Casanova est devenue la femme-phare de la Résistance communiste au féminin.

12. Pour une discussion plus approfondie de l'occultation des femmes et de la notion de « résistance », voir Paula Schwartz, « Redefining Resistance : Women's Activism in Wartime France ", in Margaret Randolph Higonnet et al., eds., Behind the Lines : Gender and the Two World Wars, New Haven, Yale University Press, 1987, pp. 141-153.

13. Marie-Louise Coudert, elles, la résistance, Paris, Messidor Temps Actuels, 1983. Une section entière de l'UFF, Les femmes dans la Résistance, est consacrée à la spécificité de l'activité féminine.

14. Ces formes de « brouillage » sont examinées dans Paula Schwartz, «"Partisanes" and Gender Politics in Vichy France », French Historical Studies 16:1 (Spring 1989), pp. 126-151.

15. Alain Brossat traite la collaboration féminine (imaginée ou véridique) aussi bien que ses représentations et ses enjeux, in Les tondues : un carnaval moche, Paris, Editions Manya, 1992.

16. Claudia Koonz, Mothers in the Fatherland: Women, the Family and Nazi Politics, New York, St. Martin's Press, 1987 ; Victoria De Grazia, How Fascism Ruled Women : Italy, 1922-1945, Berkeley, Los Angeles, London, University of California Press, 1992.

17. Michèle Bordeaux, « Femmes hors d'Etat français, 1940-1944 », in Rita Thalmann, ed., Femmes et Fascismes, Editions Tierce, 1986, pp. 135-155. Voir aussi les travaux de Miranda Pollard, notamment sa thèse de doctorat, "'Femme, Famille, France' : Vichy and the Politics of Gender, 1940-1944 », Trinity College, Dublin, 1989, qui paraîtra sous le titre (provisoire) de New Women and New Men : Sexual Politics in Vichy France, 1940-1944. Des 771 pages consacrées à l'état actuel des travaux historiographiques de la France de Vichy, seules huit portent sur sa politique misogyne : Miranda Pollard, « La politique du 
travail féminin ", in Jean-Pierre Azéma et François Bédarida, eds., Vichy et les Français, Paris, Fayard, 1992, pp. 242-250. Enfin, voir l'excellente mise au point d'Hélène Eck, «Les Françaises sous Vichy », in Françoise Thébaud, ed., Histoire des femmes en Occident, vol. 5, Le XXe siècle, Paris, Plon, 1992, pp. 185-211.

18. Jacques Semelin fait le point de tous les éléments structurants de la Résistance, à l'exception de celui de la différence des sexes. "Qu'est-ce que "résister" ? ", Esprit n 198, Janvier 1994, pp. 50-63.

19. P. Schwartz, «'Partisanes' and Gender Politics... », op. cit.

20. Borras figure sous le nom de Charua dans Charlotte Delbo, Le Convoi du 24 janvier, Paris, Editions de Minuit, 1965, pp. 67-69.

21. L'exception notable est la Bibliothèque Marguerite Durand à Paris, qui est spécialisée en histoire des femmes et du féminisme.

22. Pour la France de Vichy, cette politique touchait surtout aux domaines du travail, de l'éducation, et de la famille. Miranda Pollard, « Women and the National Revolution ", in Roderick Kedward and Roger Austin, eds., Vichy France and the Resistance: Culture and Ideology, Totowa, New Jersey, Barnes \& Noble, 1985, pp. 36-47. 23. L'article de Gisela Bock fait date: « Racism and Sexism in Nazi Germany : Motherhood, Compulsory Sterilization, and the State », in Renate Bridenthal et al., eds., When Biology Became Destiny : Women in Weimar and Nazi Germany, New York, Monthly Review Press, 1984, pp. 271-296. Une version abrégée est parue en français : « Racisme, stérilisation obligatoire et maternité sous le national-socialisme ", in Rita Thalmann, ed., Femmes et fascismes, Paris, Editions Tierce, 1986, pp. 99-115.

24. H. R. Kedward, Resistance in Vichy France: A Study of Ideas and Motivations in the Southern Zone, 1940-42, Oxford, Oxford University Press, 1978 ; John F. Sweets, Choices in Vichy France : The French under Nazi Occupation, Oxford, Oxford University Press, 1986.

25. L'activité résistante des femmes qui ont « secondé » leurs maris ressort aisément de biographies qu'elles ont consacrées à leurs époux. Gilberte Brossolette, Il s'appelait Pierre Brossolette, Paris, Albin-Michel, 1976 ; Simone Martin-Chauffier, A bientôt quand même, Paris, Calmann-Lévy, 1976.

26. Paula Schwartz, « Une répression peut engendrer une autre : mémoire, politiques de répression, et femmes communistes, 1940-1944 », Cahiers de l'IHTP, « Identités féminines et violences politiques, 1900-1985 ", à paraître.

27. Le Silence de la mer, écrit en 1941 par Vercors (Jean Bruller), a été édité par les Editions de Minuit et distribué dans la clandestinité à partir de 1942.

28. Paula Schwartz, «Women, Resistance, and the Politics of Representation in France : The Case of Le Silence de la mer ", communication inédite, Berkshire Conference on the History of Women, June 1990.

29. Chabrol met en scène l'histoire de Marie-Louise Giraud (Marie Latour dans le film), l'avorteuse qui fut exécutée à Paris en 1943. Francis Szpiner a reconstitué son histoire à partir de son dossier du Tribunal d'État : Une Affaire de femmes, Paris, Editions Balland, 1986.

30. Miranda Pollard, «Vichy, femmes et représentations », communication à Féminismes et Nazisme : Colloque en hommage à Rita Thalmann, décembre 1992, à paraître. Je remercie l'auteur, qui a bien voulu me communiquer un exemplaire de son texte. Voir aussi l'analyse du Chagrin et la Pitié par Stan Reynolds, « Un train peut en cacher un autre ", in Projets féministes, n 3, octobre 1994.

31. Virginia Woolf, Three Guineas, New York, Harcourt Brace Jovanovich, 1938. 
32. Les paradoxes nombreux de la citoyenneté des femmes en France sont explorés par Geneviève Fraisse: «Quand gouverner n'est pas représenter », Esprit, nº 198 (janvier 1994), pp. 103-114.

33. Siân Reynolds, « Marianne's Citizens ? Women, the Republic and Universal Suffrage in France ", in Siân Reynolds, ed., Women, State and Revolution : Essays on Power and Gender in Europe since 1789, Amherst, The University of Massachusetts Press, 1987, pp. 102-122. Pour la génération de la guerre, voir Claire Duchen, Women's Rights and Women's Lives in France, 1944-1968, London, Routledge, 1994, particulièrement le chapitre intitulé «Liberation ", pp. 7-32.

34. Voir le numéro spécial de Pénélope, « Mémoires de femmes ", n 12 (printemps 1985), surtout la contribution de Dominique Veillon, « Résister au féminin », pp. 87-92.

\section{RÉSUMÉS}

La notion de « différence des sexes » est porteuse de nouveaux enseignements sur les formes et le fonctionnement de la Résistance. Du rôle des femmes dans la Résistance à la construction de comportements dits «masculins" et "féminins", les problématiques, approches et objets d'étude ont évolué dans le demi siècle depuis la fin de la Deuxième Guerrre mondiale. Le caractère sexué des rôles, des moyens de lutte et de la mémoire résistante découle en grande partie de leur contexte social et politique, hautement structuré au préalable par la différence des sexes. Des problèmes conceptuels et épistémologiques restent à affronter, tels la définition de « résistance » et la catégorie universalisée de « femmes ».

Paula Schwartz: Gender Differences in the Resistance

Gender is a category of analysis which enables us to « see " the French Resistance in news ways. From the role of women in the movement to the social construction of so-called «male » and female " behavior : questions and approaches pertaining to the study of women in the French Resistance have evolved in the half-century since the close of the Second World War. The gendered nature of political roles, strategies, and memory of resistance derives in large part from the gendered universe in which men and women lived their daily lives. Persistent conceptual and epistemological problems, such as the definition of « resistance ", and the universaling category of « women ", continue to affect what and how we see.

\section{AUTEUR}

\section{PAULA SCHWARTZ}

Paula SCHWARTZ historienne et professeur d'études françaises à Middlebury College (Vermont, USA). Ses recherches (thèse et articles) sont consacrées aux femmes, au parti communiste français et à la Résistance. Son ouvrage en cours, Gender, Politics and Memory, traite de la différence des sexes dans la Résistance à travers une micro-histoire de la manifestation de la rue de Buci (mai 1942). 